\title{
COUNTEREXAMPLE OF LOSS OF REGULARITY FOR FRACTIONAL ORDER EVOLUTION EQUATIONS WITH DEGENERATING AND OSCILLATING COEFFICIENTS
}

\author{
XIAOJUN LU
}

1. Basque Center for Applied Mathematics(BCAM)

Alameda de Mazarredo 14, 48009 Bilbao, Bizkaia, Spain

\begin{abstract}
For weak evolution models of fractional order with singularity near the origin, the joint influence from the principal $\sigma$-Laplacian operator, degenerating part and oscillating part is of prime concern in the discussion of regularity behavior of the solutions. We apply the sophisticated techniques from microlocal analysis to explore the upper bound of loss of regularity. Furthermore, in order to demonstrate the optimality of the estimates, a delicate counterexample with periodic coefficients will be constructed to show the lower bound of loss of regularity by the application of Fourier analysis and instability arguments. This optimality discussion develops the theory in 4] 5] 19 20] by combining both oscillation and degeneracy of the coefficients.
\end{abstract}

RÉsumÉ. Pour les modèles d'évolution d'ordre fractionnaire faibles avec la singularité près de l'origine, l'influence conjointe d'opérateur principal de $\sigma$-Laplace, partie dégénérée et partie oscillante est notre préoccupation dans la discussion sur le comportement de la régularité des solutions. D'une part, ici nous appliquons les techniques sophistiquées de l'analyse micro-locale à explorer la limite supérieure de la perte de régularité. Et d'autre part, afin de démontrer l'optimalité des estimations, les contre-exemples délicats avec coefficients périodiques seront construits pour montrer la borne inférieure de la perte de régularité en appliquant l'analyse de Fourier et l'argument d'instabilité. Cette discussion d'optimalité développe la théorie de 4 5] 20] en combinant l'oscillation et la dégénérescence des coefficients.

\section{Introduction}

Pseudodifferential operators, especially fractional order operators (also called Riesz fractional derivatives) are very important mathematical models which describe plenty of anomalous dynamic behaviors in our daily life, such as charge carrier transport in amorphous semiconductors, nuclear magnetic resonance diffusometry in percolative and porous media, transport on fractal geometries, diffusion of a scalar tracer in an array of convection rolls, dynamics of a bead in a polymeric network, transport in viscoelastic materials, etc. More interesting industrial applications and modeling processes please refer to [15] [16] [17] [22].

First we recall the regularity behavior of the evolutional operator on $[0, T] \times \mathbb{R}, \mathscr{L}=\partial_{t}^{2}-\lambda^{2}(t) \partial_{x}^{2}$, where $\lambda(t)$ is the measure function of degeneracy, which is defined as follows.

- Let $\Lambda(t) \triangleq \int_{0}^{t} \lambda(\tau) d \tau$, a measure function of degeneracy $\lambda(t) \in C^{2}$ is a positive function satisfying:

$$
\lambda(0)=0, \quad \lambda^{\prime}(t)>0, \quad \frac{\lambda^{\prime}(t)}{\lambda(t)} \sim \frac{\lambda(t)}{\Lambda(t)}, \quad\left|\lambda^{\prime \prime}(t)\right| \lesssim \lambda(t)\left(\frac{\lambda(t)}{\Lambda(t)}\right)^{2} .
$$

It is worth noticing that the above model precisely generalizes the weakly hyperbolic operators with infinitely/finitely degenerating coefficients [1]29. By applying the diagonalization techniques introduced in 6 . while considering the propagation of mild singularities for semi-linear weakly hyperbolic equations, we know the fact that there exists no loss of regularity for this kind of operator.

Another address of the author is Department of Mathematics, Zhejiang University, 310027 Hangzhou, P. R. China. Corresponding author: lvxiaojun1119@hotmail.de.

AMS 2010 subject classification: 35L10, 35L15, 35L80, 35L90, 35S10.

Key words and phrases: Fourier analysis, weak $\sigma$-evolution equations, micro-local analysis, principal symbols, loss of regularity, difference of regularity of initial Cauchy data, normal form diagonalization, instability argument.

Mots clés et phrases: Analyse de Fourier, $\sigma$-équations d'évolution faibles, l'analyse micro-locale, symboles principaux, la perte de régularité, la différence de régularité des données initiales de Cauchy, forme normale par diagonalisation, l'argument de l'instabilité. 
In order to consider the impact of oscillation on the regularity behavior from the principal elliptic operator, we introduced in 20] a brand-new weakly hyperbolic operator with both oscillating and degenerating coefficients on $[0, T] \times \mathbb{R}: \mathscr{L}=\partial_{t}^{2}-\lambda^{2}(t) b^{2}(t) \partial_{x}^{2}$, where $\lambda(t)$ is the measure function of degeneracy, $b(t) \in C^{2}(0, T]$ describes the oscillation of the principal elliptic operator near the origin 0. More precisely,

- $b_{0} \triangleq \inf _{t \in(0, T]} b(t) \leq b(t) \leq b_{1} \triangleq \sup _{t \in(0, T]} b(t), \quad b_{0}, b_{1}>0$;

- $\left|b^{(k)}(t)\right| \leq C\left(\frac{\lambda(t)}{\Lambda(t)} \nu(t)\right)^{k}, C>0, k=1,2$, where $\nu(t)$ is a measure function of oscillation, which is defined as a continuous and decreasing positive function on a finite time interval.

By two steps of diagonalization procedure, we have an insightful understanding of the impact from the oscillating coefficients. Detailed description of both the loss of regularity and difference of regularity of the initial Cauchy data are given in 20. In previous literature 6, 11, 12, 13, 14, 18, 20, one mainly discussed the wave equation with lower order terms. As a matter of fact, the finite propagation speed holds for this kind of operator. In reality, most of the operators have infinite propagation speed, such as heat equation $u_{t}-\Delta u=0$, Petrowsky equation $u_{t t}+\Delta^{2} u=0$, etc. In this manuscript, the weak evolution operator of fractional order with oscillating and degenerating coefficients, which has infinite propagation speed, is of prime concern:

$$
\mathscr{L}=\partial_{t}^{2}+A_{0}(t, \sqrt{-\Delta})
$$

where

$$
A_{0}(t, \sqrt{-\Delta}) \triangleq \lambda^{2}(t) b^{2}(t)(-\Delta)^{\sigma}
$$

with $\sigma>1$ and $(-\Delta)^{\sigma}$ defined on the torus $\mathbb{T}^{N}$. In this model, we call $A_{0}(t, \sqrt{-\Delta})$ the principal part in the sense of Petrowsky. One typical example of the coefficients on the principal part is $b(t)=2+\sin \left((\log (1 / t))^{\kappa}\right)$, $\kappa \in(1, \infty)$, which satisfies the assumptions with $\nu(t)=(\log (1 / t))^{\kappa-1}$. Up to now, there is still no complete conclusion about Levi-condition with oscillation 12 . However, the theory of pseudodifferential operators assures the existence and uniqueness of the solution for the Cauchy problem of (1). As an important application of this model, actually, through Nirenberg's transformation $v=1-\exp (-u)$, the problem of the semi-linear Cauchy problem $u_{t t}-a^{2}(t) \Delta u=u_{t}^{2}-a^{2}(t)|\nabla u|^{2}$ can be turned into the linear problem $v_{t t}-a^{2}(t) \Delta v=0$. More discussion in this respect please refer to [8]. In the following, we explore carefully the joint influence upon the regularity behavior of (1) from both oscillation and degeneracy of the principal elliptic operator $A_{0}(t, \sqrt{-\Delta})$.

Under the above assumptions of $\lambda(t)$ and $b(t)$, one has the following regularity statement:

Theorem 1.1. Let us consider the Cauchy problem of model (1) on $[0, T] \times \mathbb{T}^{N}$,

$$
\mathscr{L} u=0, u(0, x)=u_{0}(x), u_{t}(0, x)=u_{1}(x) .
$$

If the initial Cauchy data satisfy

$$
u_{0} \in H^{s}\left(\mathbb{T}^{N}\right), \quad u_{1} \in \frac{1}{\Lambda^{-1}\left(\frac{2^{P_{1}}}{(\sqrt{1-\Delta})^{\sigma}}\right)} H^{s}\left(\mathbb{T}^{N}\right),
$$

where $P_{1} \in \mathbb{N}_{+}$is a fixed constant and the Sobolev index $s$ is sufficiently large, then there exists a unique solution $u$ in the following function spaces:

$$
\begin{gathered}
u \in C\left([0, T], \exp \left(C_{\alpha} \nu\left(\left(\frac{\Lambda}{\nu}\right)^{-1}\left(\frac{2^{P_{2}}}{(\sqrt{1-\Delta})^{\sigma}}\right)\right)\right) H^{s}\left(\mathbb{T}^{N}\right)\right), \\
u_{t} \in C\left([0, T], \exp \left(C_{\alpha} \nu\left(\left(\frac{\Lambda}{\nu}\right)^{-1}\left(\frac{2^{P_{2}}}{(\sqrt{1-\Delta})^{\sigma}}\right)\right)\right) H^{s-\sigma}\left(\mathbb{T}^{N}\right)\right) ;
\end{gathered}
$$

where $C_{\alpha} \in \mathbb{R}_{+}$and $P_{2} \in \mathbb{N}_{+}$are fixed constants. In this theorem, $\Lambda^{-1}$ and $\left(\frac{\Lambda}{\nu}\right)^{-1}$ denote respectively the corresponding inverse functions. In fact, according to the monotonicity of $\lambda(t)$ and $\nu(t)$, both inverse functions are well-defined.

Proof. Similar as in 20, the above theorem can be proved by applying comparison lemma and two steps of diagonalization procedure. Here we do not go to details. Interested readers please refer to [16 [17. Q. E. D. 
COUNTEREXAMPLE OF LOSS OF REGULARITY FOR FRACTIONAL ORDER EVOLUTION EQUATIONS WITH DEGENERATING AND OSCILLATIN

In the following, we give some typical examples to explain the different influence from various kinds of degenerating and oscillating coefficients. First we consider the influence of degenerating coefficients for the Cauchy problem

$$
u_{t t}+\lambda^{2}(t)(-\Delta)^{\sigma} u=0, \quad u(0, x)=u_{0}(x), \quad u_{t}(0, x)=u_{1}(x)
$$

According to Theorem 1.1, one has

- $\lambda(t)=\frac{1}{\log [n] \frac{1}{t}}, n \geq 1,($ Logarithmically degenerating coefficient)

$$
\left(u_{0}, u_{1}\right) \in\left(H^{s} \times \frac{1}{\Lambda^{-1}\left(\frac{2^{P_{1}}}{(\sqrt{1-\Delta})^{\sigma}}\right)} H^{s}\right) \Rightarrow u \in C\left([0, T], H^{s}\right) \bigcap C^{1}\left([0, T], H^{s-\sigma}\right),
$$

where $\Lambda(t)=O\left(\frac{t}{\log [n] \frac{1}{t}}\right)$;

- $\lambda(t)=t^{\ell}, \ell \geq 1$, (Finitely degenerating coefficient)

$$
\left(u_{0}, u_{1}\right) \in\left(H^{s} \times H^{s-\frac{\sigma}{\ell+1}}\right) \Rightarrow u \in C\left([0, T], H^{s}\right) \bigcap C^{1}\left([0, T], H^{s-\sigma}\right) ;
$$

- $\lambda(t)=\frac{1}{t^{2}} \exp \left(-\frac{1}{t}\right)$, (Infinitely degenerating coefficient)

$$
\left(u_{0}, u_{1}\right) \in\left(H^{s} \times \sigma \log (\sqrt{1-\Delta}) H^{s}\right) \Rightarrow u \in C\left([0, T], H^{s}\right) \bigcap C^{1}\left([0, T], H^{s-\sigma}\right) ;
$$

- $\lambda(t)=\frac{d}{d t} \exp \left(-\exp ^{[n]}\left(\frac{1}{t}\right)\right), n \geq 1$, (Super infinitely degenerating coefficient)

$$
\left(u_{0}, u_{1}\right) \in\left(H^{s} \times \log ^{[n]+1}(\sqrt{1-\Delta})^{\sigma} H^{s}\right) \Rightarrow u \in C\left([0, T], H^{s}\right) \bigcap C^{1}\left([0, T], H^{s-\sigma}\right) .
$$

Next we show the influence of oscillating coefficients for the Cauchy problem

$$
u_{t t}+\lambda^{2}(t) b^{2}(t)(-\Delta)^{\sigma} u=0, \quad u(0, x)=u_{0}(x), \quad u_{t}(0, x)=u_{1}(x) .
$$

Suppose that the initial Cauchy data satisfy

$$
\left(u_{0}, u_{1}\right) \in\left(H^{s} \times \frac{1}{\Lambda^{-1}\left(\frac{2^{P_{1}}}{(\sqrt{1-\Delta})^{\sigma}}\right)} H^{s}\right),
$$

then according to Theorem 1.1, we have: $\left(C_{\alpha} \in \mathbb{R}_{+}\right)$

- No loss of derivatives, $\nu(t) \sim 1$,

$$
u \in C\left([0, T], H^{s}\right), \quad u_{t} \in C\left([0, T], H^{s-\sigma}\right) ;
$$

- Finite loss of derivatives, $\nu(t)=\log \frac{1}{\Lambda(t)}$,

$$
u \in C\left([0, T],(\sqrt{1-\Delta})^{\sigma C_{\alpha}} H^{s}\right), \quad u_{t} \in C\left([0, T],(\sqrt{1-\Delta})^{\sigma C_{\alpha}} H^{s-\sigma}\right) ;
$$

- Arbitrarily small loss of derivatives, $\nu(t)=\left(\log \frac{1}{\Lambda(t)}\right)^{\gamma}, \gamma \in(0,1)$,

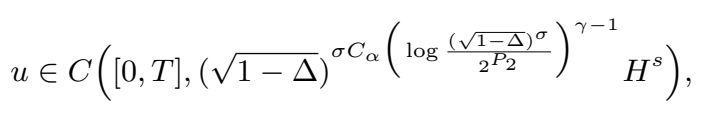

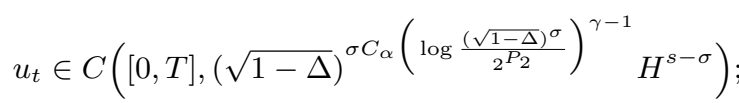

- Infinite loss of derivatives,

$$
\begin{aligned}
& \nu(t)=\left(\log \frac{1}{\Lambda(t)}\right)\left(\log ^{[2]} \frac{1}{\Lambda(t)}\right)^{\gamma_{2}} \cdots\left(\log ^{[n]} \frac{1}{\Lambda(t)}\right)^{\gamma_{n}}, \gamma_{i} \in(0,1], i=2, \cdots, n \\
& u \in C\left([0, T],(\sqrt{1-\Delta})^{\sigma C_{\alpha}}\left(\log [2] \frac{(\sqrt{1-\Delta})^{\sigma}}{2^{P_{2}}}\right)^{\gamma_{2}} \cdots\left(\log [n] \frac{(\sqrt{1-\Delta})^{\sigma}}{2^{P_{2}}}\right)^{\gamma_{n}} H^{s}\right), \\
& u_{t} \in C\left([0, T],(\sqrt{1-\Delta})^{\sigma C_{\alpha}}\left(\log ^{[2]} \frac{(\sqrt{1-\Delta})^{\sigma}}{2^{P_{2}}}\right)^{\gamma_{2}} \cdots\left(\log ^{[n]} \frac{(\sqrt{1-\Delta})^{\sigma}}{2^{P_{2}}}\right)^{\gamma_{n}} H^{s-\sigma}\right) \text {. }
\end{aligned}
$$


We call all the above losses incurred by the oscillation measure function $\nu$-loss of derivatives. In the following, we construct a delicate counterexample to demonstrate the existence of $\nu$-loss when the infinite propagation speed appears. Related topics please refer to [2] 3] 11] 13] 14] 24].

Let us consider the following Cauchy problem on $[0, T] \times \mathbb{T}$

$$
\partial_{t}^{2} u+\lambda^{2}(t) b^{2}(t)\left(-\partial_{x}^{2}\right)^{\sigma} u=0, \quad u(0, x)=u_{0}(x), \quad \partial_{t} u(0, x)=u_{1}(x),
$$

with $2 \pi$-periodic initial Cauchy data $u_{0}, u_{1}$. Actually, on the torus $\mathbb{T}^{N}$, one can apply the same procedure.

Definition 1.2. For a $2 \pi$-periodic solution $u=u(t, x)$ in the $x$-variable, we introduce the homogeneous energy

$$
\dot{\mathbb{E}}_{s}(u)(t) \triangleq \lambda^{2}(t)\|u(t, \cdot)\|_{\dot{H}^{s}(\mathbb{T})}^{2}+\left\|\partial_{t} u(t, \cdot)\right\|_{\dot{H}^{s-\sigma}(\mathbb{T})}^{2}, \quad s>\sigma,
$$

where $\dot{H}^{s}(\mathbb{T})$ denotes the homogeneous Sobolev space of exponent $s$ on the torus $\mathbb{T}=\mathbb{R} / 2 \pi \mathbb{Z}$.

Actually, Theorem 1.1. indicates the following conclusion which shows at most a $\nu$-loss.

Corollary 1.3. Let us consider a family of Cauchy problems on $[0, T] \times \mathbb{T}$,

$$
\partial_{t}^{2} u^{k}+\lambda^{2}(t) b_{k}^{2}(t)\left(-\partial_{x}^{2}\right)^{\sigma} u^{k}=0, u^{k}(0, x)=u_{0}^{k}(x), \partial_{t} u^{k}(0)=u_{1}^{k}(x) .
$$

Define $\mu(s)$ as $\Lambda(s) / \nu(s)$ and $\left\{b_{k}\right\}_{k}$ satisfy all the assumptions in Theorem 1.3. with constants independent of $k$. If the initial Cauchy data satisfy

$$
u_{0}^{k} \in H^{s}(\mathbb{T}), \quad u_{1}^{k} \in \frac{1}{\Lambda^{-1}\left(\frac{2^{P_{1}}}{(\sqrt{1-\Delta})^{\sigma}}\right)} H^{s}(\mathbb{T}),
$$

where $P_{1} \in \mathbb{N}_{+}$is a fixed constant, then there exists a unique solution $u^{k}$ in the following function spaces:

$$
\begin{gathered}
u^{k} \in C\left([0, T] ; \exp \left(C_{\alpha} \nu\left(\mu^{-1}\left(2^{P_{2}} /(\sqrt{1-\Delta})^{\sigma}\right)\right) H^{s}(\mathbb{T})\right),\right. \\
u_{t}^{k} \in C\left([0, T] ; \exp \left(C_{\alpha} \nu\left(\mu^{-1}\left(2^{P_{2}} /(\sqrt{1-\Delta})^{\sigma}\right)\right)\right) H^{s-\sigma}(\mathbb{T})\right),
\end{gathered}
$$

with positive constants $C_{\alpha}$ and $P_{2} \in \mathbb{N}_{+}$is a fixed constant. Moreover, $\mu^{-1}$ denotes the inverse function of $\mu$.

Now we are ready to introduce the main theorem of this paper, which shows that the $\nu$-loss of derivatives really appears. For convenience's sake, we only prove the the case for homogeneous energy. Similarly, one can define the non-homogeneous energy as

$$
\mathbb{E}_{s}(u)(t) \triangleq \lambda^{2}(t)\|u(t, \cdot)\|_{H^{s}(\mathbb{T})}^{2}+\left\|\partial_{t} u(t, \cdot)\right\|_{H^{s-\sigma}(\mathbb{T})}^{2}, \quad s>\sigma,
$$

where $H^{s}(\mathbb{T})$ denotes the standard Sobolev space of exponent $s$ on the torus $\mathbb{T}=\mathbb{R} / 2 \pi \mathbb{Z}$. And the result also holds for the inhomogeneous energy case. This will be explained during the proof.

Theorem 1.4. For the Cauchy problem (4), there exists

- a sequence of coefficients $\left\{b_{k}(t)\right\}_{k}$ satisfying all assumptions of Theorem 1.1 with constants independent of $k$;

- a sequence of initial Cauchy data $\left\{\left(u_{0}^{k}(x), u_{1}^{k}(x)\right)\right\}_{k} \in \dot{H}^{s}(\mathbb{T}) \times \frac{1}{\Lambda^{-1}\left(\frac{2^{P}}{(\sqrt{1-\Delta})^{\sigma}}\right)} \dot{H}^{s}(\mathbb{T})$;

such that the sequence of corresponding solutions $\left\{u^{k}(t, x)\right\}_{k}$ from $C^{\infty}([0, T] \times \mathbb{T})$ satisfies

$$
\begin{gathered}
\sup _{k} \dot{\mathbb{E}}_{\sigma}\left(u^{k}\right)(0) \leq C(\varepsilon), \\
\sup _{k} \dot{\mathbb{E}}_{\sigma}\left(\exp \left(-c_{1}(\varepsilon) \nu\left(\mu^{-1}\left(2^{P_{2}} /(\sqrt{1-\Delta})^{\sigma}\right)\right)\right) u^{k}\right)(t)=+\infty, \text { for any } t \in(0, T],
\end{gathered}
$$

where $C(\varepsilon)$ and $c_{1}(\varepsilon)$ depending on the sufficiently small positive constant $\varepsilon$.

The rest of the paper is organized as follows. Section 2 is a brief introduction of pseudodifferential operators on the torus. In Section 3, we discuss the optimality of the statement for general $\sigma>1$ on the torus $\mathbb{T}$ by the application of instability argument, which develops the discussion in [5] by adding degenerating coefficients. This is an important complement of the discussion in $[26$ for $\sigma \in(0,1]$, in which case, the finite propagation speed holds. 


\section{Prerequisite: A Brief review of PdOs on the torus}

In this section we mainly recall the pseudodifferential operators (PDOs) defined on the torus $\mathbb{T}^{N}$, which is a typical compact smooth manifold without boundary. The discussion can be applied to general LaplaceBeltrami operators successfully, such as on the sphere $\mathbb{S}^{N}$, etc. First and foremost, we give the precise definition of PDOs on the torus.

Definition 2.1. On the torus $\mathbb{T}^{N}$, let $u \in C^{\infty}\left(\mathbb{T}^{N}\right)$, then the sequence of Fourier series $\{\hat{u}(m)\}_{m \in \mathbb{Z}^{N}}$ defined by

$$
\hat{u}(m) \triangleq(2 \pi)^{-N} \int_{\mathbb{T}^{N}} u(x) \exp (-i\langle m, x\rangle) d x,
$$

is a rapidly decreasing sequence.(25]) By duality, we may produce an extension to the periodic distributions:

$$
\mathcal{F}: \mathscr{D}^{\prime}\left(\mathbb{T}^{N}\right) \rightarrow \mathscr{S}^{\prime}\left(\mathbb{Z}^{N}\right), \quad \mathcal{F}^{-1}: \mathscr{S}^{\prime}\left(\mathbb{Z}^{N}\right) \rightarrow \mathscr{D}^{\prime}\left(\mathbb{T}^{N}\right)
$$

With the Fourier transform, we define a generalized linear pseudodifferential operator

$$
F(\sqrt{-\Delta}): D(F(\sqrt{-\Delta})) \subset L^{2}\left(\mathbb{T}^{N}\right) \rightarrow L^{2}\left(\mathbb{T}^{N}\right)
$$

as

$$
F(\sqrt{-\Delta}) u(x) \triangleq \sum_{m \in \mathbb{Z}^{N}} F(|m|) \mathcal{F} u(m) \exp (i\langle m, x\rangle) .
$$

The sequence $\left\{F(|m|): m \in \mathbb{Z}^{N}\right\}$ is referred to as the torus symbol of $F(\sqrt{-\Delta})$, which is also a polynomially bounded sequence. Furthermore, when $F=|x|^{s}$, then one has the homogeneous Sobolev space $\dot{H}^{s}\left(\mathbb{T}^{N}\right)$. While when $F=\left(1+|x|^{2}\right)^{\frac{s}{2}}$, one has the inhomogeneous Sobolev space $H^{s}\left(\mathbb{T}^{N}\right)$.

Lemma 2.2. The RHS of (11) converges in the distributional sense. Moreover, when $F$ is a real-valued functional, then the operator $F(\sqrt{-\Delta})$ in Definition 2.1 is a self-adjoint operator.

Proof. (I) Actually, in Definition 2.1, $F(\sqrt{-\Delta})$ is defined in the distributional sense. Indeed, for $\forall \eta \in \mathscr{D}\left(\mathbb{T}^{N}\right)$, since $-\Delta\left(\mathscr{D}\left(\mathbb{T}^{N}\right)\right)=\mathscr{D}\left(\mathbb{T}^{N}\right)$, then there exists a unique $\eta_{k} \in \mathscr{D}\left(\mathbb{T}^{N}\right)$ such that $\underbrace{-\Delta \cdots-\Delta}_{k} \eta=\eta_{k}$ for each $k \in \mathbb{N}$. As a result,

$$
\begin{aligned}
& \left(\exp (i\langle m, x\rangle), \eta_{k}\right)_{L^{2}\left(\mathbb{T}^{N}\right)}=(\exp (i\langle m, x\rangle), \underbrace{-\Delta \cdots-\Delta}_{k} \eta)_{L^{2}\left(\mathbb{T}^{N}\right)} \\
= & \left.(-\Delta \exp (i\langle m, x\rangle), \underbrace{-\Delta \cdots-\Delta}_{k-1} \eta)_{L^{2}\left(\mathbb{T}^{N}\right)} \cdots\right|_{k-1} ^{-\Delta \cdots}(\exp (i\langle m, x\rangle), \underbrace{-\Delta \cdots-\Delta}_{L^{2}\left(\mathbb{T}^{N}\right)} \\
= & |m|^{2}(\exp (i\langle m, x\rangle), \eta)_{L^{2}\left(\mathbb{T}^{N}\right)} .
\end{aligned}
$$

Hölder's inequality tells that

$$
\left|\left(\exp (i\langle m, x\rangle), \eta_{k}\right)_{L^{2}\left(\mathbb{T}^{N}\right)}\right| \leq\|\exp (i\langle m, x\rangle)\|_{L^{2}\left(\mathbb{T}^{N}\right)}\left\|\eta_{k}\right\|_{L^{2}}=\sqrt{\int_{\mathbb{T}^{N}} 1 d x\left\|\eta_{k}\right\|_{L^{2}\left(\mathbb{T}^{N}\right)}} .
$$

As a result, $\left\{\left|(\exp (i\langle m, x\rangle), \eta)_{L^{2}\left(\mathbb{T}^{N}\right)}\right|\right\}_{m}$ is a rapidly decreasing sequence w. r. p. to $m$. On the other hand, $\{\hat{u}(m)\}_{m}$ is a polynomially bounded sequence w. r. p. to $m$. Since $F$ is also a polynomially bounded function, consequently, the series on the RHS converges. i.e.

$$
\sum_{m \in \mathbb{Z}^{N}} F(|m|) \mathcal{F} u(m)(\exp (i\langle m, x\rangle), \eta)_{L^{2}\left(\mathbb{T}^{N}\right)}<\infty .
$$


(II) Let $u, v \in D(F(\sqrt{-\Delta}))$, then apply Definition 2.1, and one has

$$
\begin{aligned}
(F(\sqrt{-\Delta}) u, v)_{L^{2}\left(\mathbb{T}^{N}\right)} & =\left(\sum_{m \in \mathbb{Z}^{N}} F(|m|) \mathcal{F} u(m) \exp (i\langle m, x\rangle), \sum_{n \in \mathbb{Z}^{N}} \mathcal{F} v(n) \exp (i\langle n, x\rangle)\right)_{L^{2}\left(\mathbb{T}^{N}\right)} \\
& =\sum_{m \in \mathbb{Z}^{N}}(F(|m|) \mathcal{F} u(m) \exp (i\langle m, x\rangle), \mathcal{F} v(m) \exp (i\langle m, x\rangle))_{L^{2}\left(\mathbb{T}^{N}\right)} \\
& =\left(\sum_{m \in \mathbb{Z}^{N}} \mathcal{F} u(m) \exp (i\langle m, x\rangle), \sum_{n \in \mathbb{Z}^{N}} F(|n|) \mathcal{F} v(n) \exp (i\langle n, x\rangle)\right)_{L^{2}\left(\mathbb{T}^{N}\right)} \\
& =(u, F(\sqrt{-\Delta}) v)_{L^{2}\left(\mathbb{T}^{N}\right) .}
\end{aligned}
$$

Q. E. D.

Remark 2.3. As a matter of fact, the spectrum of Laplace-Beltrami operator $\Delta_{T}$ on the torus $\mathbb{T}^{N}$ is $\left\{0,-1^{2},-2^{2},-3^{2}, \cdots\right\}$. And the associated orthonormal basis for $L^{2}\left(\mathbb{T}^{N}\right)$ is

$$
\left\{\left(\int_{\mathbb{T}^{N}} 1 d x\right)^{-\frac{1}{2}} \exp (i\langle m, x\rangle):|m|=0,1,2,3, \cdots\right\} .
$$

And in contrast, the spectrum of Laplace-Beltrami operator $\Delta_{S}$ on the unit sphere $\mathbb{S}^{N}$ is

$$
\left\{\lambda_{k}=-k(k+N-1), k=0,1,2, \cdots\right\} .
$$

Applying Definition 2.1. of the pseudodifferential operator $\sqrt{-\partial_{x}^{2}}$ on the compact manifold $\mathbb{T}$, we obtain the following lemma.

Lemma 2.4. For $a \in \mathbb{Z}, x \in \mathbb{T}$ and a continuous function $F: \mathbb{R} \rightarrow \mathbb{R}$, one has

$$
F\left(\sqrt{-\partial_{x}^{2}}\right) \exp (i|a| x)=F(|a|) \exp (i|a| x) .
$$

Proof. For $u(x)=\exp (i|a| x), a \in \mathbb{Z}$, the Fourier series expansion is

$$
u(x)=\sum_{k \in \mathbb{Z}} \hat{u}(k) \exp (i k x),
$$

where

$$
\hat{u}(k)=(2 \pi)^{-1} \int_{\mathbb{T}} u(x) \exp (-i k x) d x= \begin{cases}1, & k=|a| \\ 0, & k \neq|a|\end{cases}
$$

Then apply the definition of pseudodifferential operators on the compact manifold $\mathbb{T}$, and we have

$$
F\left(\sqrt{-\partial_{x}^{2}}\right) \exp (i|a| x)=\sum_{k \in \mathbb{Z}} \hat{u}(k) F(|k|) \exp (i k x)=F(|a|) \exp (i|a| x) .
$$

The statement is proved. Q. E. D.

\section{Proof of Theorem 1.4}

First we introduce some auxiliary functions and sequences.

Definition 3.1. For a sufficiently small $\varepsilon>0$, we define:

$$
\begin{gathered}
w_{\varepsilon}(t) \triangleq \sin t \exp \left(2 \varepsilon \int_{0}^{t} \psi(\tau) \sin ^{2} \tau d \tau\right), \\
a_{\varepsilon}(t) \triangleq 1-4 \varepsilon \psi(t) \sin (2 t)-2 \varepsilon \psi^{\prime}(t) \sin ^{2} t-4 \varepsilon^{2} \psi^{2}(t) \sin ^{4} t,
\end{gathered}
$$

where the real-valued non-negative smooth function $\psi$ is $2 \pi$-periodic on $\mathbb{R}$ and identically 0 in a neighborhood of 0 . And assume that $\psi$ satisfies

$$
\int_{0}^{2 \pi} \psi(\tau) \sin ^{2}(\tau) d \tau=\pi
$$

It is easy to verify the following fact after simple calculation.

Lemma 3.2. According to Definition 3.1, $a_{\varepsilon} \in C^{\infty}(\mathbb{R})$ and $w_{\varepsilon} \in C^{\infty}(\mathbb{R})$. Particularly, $w_{\varepsilon}$ is the unique solution of the following ordinary differential equation with initial data

$$
\partial_{t}^{2} w_{\varepsilon}(t)+a_{\varepsilon}(t) w_{\varepsilon}(t)=0, \quad w_{\varepsilon}(0)=0, \quad \partial_{t} w_{\varepsilon}(0)=1 .
$$


COUNTEREXAMPLE OF LOSS OF REGULARITY FOR FRACTIONAL ORDER EVOLUTION EQUATIONS WITH DEGENERATING AND OSCILLATIN

Definition 3.3. Define

$$
\begin{gathered}
\left\{\rho_{k}\right\}_{k} \triangleq\left\{2^{-P_{2}+2} \pi \frac{\Lambda\left(t_{k}\right)}{\lambda\left(t_{k}\right)} \frac{\left[\nu\left(t_{k}\right)\right]}{\nu\left(t_{k}\right)}\right\}_{k}, \\
\left\{h_{k}\right\}_{k} \triangleq\left\{2^{P_{2}} \nu\left(t_{k}\right) \frac{\lambda\left(t_{k}\right)}{\Lambda\left(t_{k}\right)}\right\}_{k}, \\
\left\{\delta_{k}\right\}_{k} \triangleq\left\{\lambda\left(t_{k}\right)\right\}_{k},
\end{gathered}
$$

where $\left\{t_{k}\right\}_{k}$ is a zero sequence satisfying

$$
\left(2^{P_{2}} \nu\left(t_{k}\right) / \Lambda\left(t_{k}\right)\right)^{\frac{1}{\sigma}} \in \mathbb{N}_{+}
$$

for each $k \in \mathbb{N} .[a]$ represents the integer part of $a$.

Definition 3.4. In addition, we introduce two time-sequences:

$$
\left\{t_{k}^{\prime}\right\} \triangleq\left\{t_{k}+\rho_{k}\right\}_{k}
$$

and

$$
\left\{t_{k}^{\prime \prime}\right\} \triangleq\left\{t_{k}-\rho_{k}\right\}_{k},
$$

and accordingly, one defines three time intervals $I_{k}, I_{k}^{\prime}$ and $I_{k}^{\prime \prime}$,

$$
\begin{aligned}
I_{k} & \triangleq\left[t_{k}-\frac{\rho_{k}}{2}, t_{k}+\frac{\rho_{k}}{2}\right], \\
I_{k}^{\prime} & \triangleq\left[t_{k}^{\prime}-\frac{\rho_{k}}{2}, t_{k}^{\prime}+\frac{\rho_{k}}{2}\right], \\
I_{k}^{\prime \prime} & \triangleq\left[t_{k}^{\prime \prime}-\frac{\rho_{k}}{2}, t_{k}^{\prime \prime}+\frac{\rho_{k}}{2}\right] .
\end{aligned}
$$

Remark 3.5. $\left\{I_{k}\right\}_{k}$ is called sequence of oscillation intervals, $\left\{I_{k}^{\prime}\right\}_{k}$ is sequence of right buffer intervals, and $\left\{I_{k}^{\prime \prime}\right\}_{k}$ is sequence of left buffer intervals.

Remark 3.6. It is easy to see that the sequences $\left\{t_{k}\right\}_{k},\left\{\rho_{k}\right\}_{k}$, tend to 0 , while the sequence $\left\{h_{k}\right\}_{k}$ tends to $+\infty$. Such choice of $\rho_{k}$ guarantees that $I_{k}$ is contained in $(0, T]$. Furthermore, $h_{k} \rho_{k} /(4 \pi),\left(h_{k} / \delta_{k}\right)^{\frac{1}{\sigma}} \in \mathbb{N}_{+}$.

We divide our proof of Theorem 1.4. into three steps.

Step 1: Construction of a sequence of oscillating coefficients

Define a monotonously increasing function $\mu \in C^{\infty}(\mathbb{R})$ as

$$
\mu(x) \triangleq \begin{cases}0, & x \in\left(-\infty,-\frac{1}{3}\right] \\ 1, & x \in\left[\frac{1}{3},+\infty\right)\end{cases}
$$

Now we introduce a family of coefficients $\left\{a_{k}=a_{k}(t)\right\}_{k}$ :

$$
a_{k}(t) \triangleq \begin{cases}\lambda^{2}(t), & t \in[0, T] \backslash\left(I_{k}^{\prime} \cup I_{k} \cup I_{k}^{\prime \prime}\right) ; \\ \delta_{k}^{2} a_{\varepsilon}\left(h_{k}\left(t-t_{k}\right)\right), & t \in I_{k} ; \\ \delta_{k}^{2}\left(1-\mu\left(\frac{t-t_{k}^{\prime}}{\rho_{k}}\right)\right)+\lambda^{2}(t) \mu\left(\frac{t-t_{k}^{\prime}}{\rho_{k}}\right), & t \in I_{k}^{\prime} ; \\ \delta_{k}^{2} \mu\left(\frac{t-t_{k}^{\prime \prime}}{\rho_{k}}\right)+\lambda^{2}(t)\left(1-\mu\left(\frac{t-t_{k}^{\prime \prime}}{\rho_{k}}\right)\right), & t \in I_{k}^{\prime \prime} .\end{cases}
$$

Consequently, the oscillating part $\left\{b_{k}(t)\right\}_{k}$ is

$$
b_{k}^{2}(t) \triangleq \begin{cases}1, & t \in[0, T] \backslash\left(I_{k}^{\prime} \cup I_{k} \cup I_{k}^{\prime \prime}\right) ; \\ \frac{\delta_{k}^{2}}{\lambda^{2}(t)} a_{\varepsilon}\left(h_{k}\left(t-t_{k}\right)\right), & t \in I_{k} ; \\ \frac{\delta_{k}^{2}}{\lambda^{2}(t)}\left(1-\mu\left(\frac{t-t_{k}^{\prime}}{\rho_{k}}\right)\right)+\mu\left(\frac{t-t_{k}^{\prime}}{\rho_{k}}\right), & t \in I_{k}^{\prime} \\ \frac{\delta_{k}^{2}}{\lambda^{2}(t)} \mu\left(\frac{t-t_{k}^{\prime \prime}}{\rho_{k}}\right)+\left(1-\mu\left(\frac{t-t_{k}^{\prime \prime}}{\rho_{k}}\right)\right), & t \in I_{k}^{\prime \prime}\end{cases}
$$


In addition, we suppose

$$
\begin{aligned}
& d_{0}^{+} \leq \inf _{k} \frac{\lambda\left(t_{k}\right)}{\lambda\left(t_{k}+\rho_{k}\right)} \leq \sup _{k} \frac{\lambda\left(t_{k}\right)}{\lambda\left(t_{k}+\rho_{k}\right)} \leq d_{1}^{+}, \\
& d_{0}^{-} \leq \inf _{k} \frac{\lambda\left(t_{k}\right)}{\lambda\left(t_{k}-\rho_{k}\right)} \leq \sup _{k} \frac{\lambda\left(t_{k}\right)}{\lambda\left(t_{k}-\rho_{k}\right)} \leq d_{1}^{-},
\end{aligned}
$$

where $d_{1}^{+}, d_{1}^{-}, d_{2}^{+}$and $d_{2}^{-}$are all positive constants. Actually, when $P_{2}$ is sufficiently large, then $t_{k}$ is always the dominating part. Interested readers can verify this fact with specific finitely or infinitely degenerating coefficients. Simple calculations lead to

$$
0<b_{0} \leq \inf _{t \in(0, T]} b_{k}(t) \leq \sup _{t \in(0, T]} b_{k}(t) \leq b_{1}<\infty,
$$

where $b_{0}$ and $b_{1}$ are independent of $k$. Moreover, in the interval $I_{k} \bigcup I_{k}^{\prime} \bigcup I_{k}^{\prime \prime}$, one has

where $C$ is independent of $k$.

$$
\left|b_{k}^{\prime}(t)\right| \leq C \frac{\lambda(t) \nu(t)}{\Lambda(t)} ; \quad\left|b_{k}^{\prime \prime}(t)\right| \leq C\left(\frac{\lambda(t) \nu(t)}{\Lambda(t)}\right)^{2},
$$

Step 2: Construction of auxiliary functions

Next we study the family of Cauchy problems in $\left[t_{k}-\rho_{k} / 2, t_{k}+\rho_{k} / 2\right] \times \mathbb{T}$,

$$
\partial_{t}^{2} u^{k}+\delta_{k}^{2} a_{\varepsilon}\left(h_{k}\left(t-t_{k}\right)\right)\left(-\partial_{x}^{2}\right)^{\sigma} u^{k}=0, u^{k}\left(t_{k}, x\right)=0, \partial_{t} u^{k}\left(t_{k}, x\right)=u_{1}^{k}(x) .
$$

Let the initial Cauchy data be

$$
u_{1}^{k}(x)=\exp \left(i\left(\frac{h_{k}}{\delta_{k}}\right)^{\frac{1}{\sigma}} x\right)
$$

and apply the coordinate transform

$$
s=h_{k}\left(t-t_{k}\right)
$$

At the same time, define

$$
v^{k}(s, x) \triangleq u^{k}(t(s), x),
$$

then for $s \in\left[-h_{k} \rho_{k} / 2, h_{k} \rho_{k} / 2\right]$, one has

$$
\partial_{s}^{2} v^{k}+\delta_{k}^{2} h_{k}^{-2} a_{\varepsilon}(s)\left(-\partial_{x}^{2}\right)^{\sigma} v^{k}=0, v^{k}(0, x)=0, \partial_{s} v^{k}(0, x)=u_{1}^{k}(x) / h_{k} .
$$

As a matter of fact, when we take Lemma 3.2. into account, then we have a unique solution for (13) in the form of

$$
v^{k}(s, x)=h_{k}^{-1} u_{1}^{k}(x) w_{\varepsilon}(s) .
$$

Transforming back to $u^{k}(t, x)$, we arrive at

$$
u^{k}(t, x)=h_{k}^{-1} \exp \left(i\left(\frac{h_{k}}{\delta_{k}}\right)^{\frac{1}{\sigma}} x\right) w_{\varepsilon}\left(h_{k}\left(t-t_{k}\right)\right)
$$

in $I_{k}$. Further calculations lead to

$$
\begin{aligned}
& u^{k}\left(t_{k}-\rho_{k} / 2, x\right)=0, \quad \partial_{t} u^{k}\left(t_{k}-\rho_{k} / 2, x\right)=\exp \left(i\left(\frac{h_{k}}{\delta_{k}}\right)^{\frac{1}{\sigma}} x\right) \exp \left(-\varepsilon \rho_{k} h_{k} / 2\right), \\
& u^{k}\left(t_{k}+\rho_{k} / 2, x\right)=0, \quad \partial_{t} u^{k}\left(t_{k}+\rho_{k} / 2, x\right)=\exp \left(i\left(\frac{h_{k}}{\delta_{k}}\right)^{\frac{1}{\sigma}} x\right) \exp \left(\varepsilon \rho_{k} h_{k} / 2\right) .
\end{aligned}
$$

Step 3: Existence of $\nu$-loss of regularity

Now we introduce an energy-increasing property in the sense of pseudo-differential operators.

Lemma 3.7. For the Cauchy problem with $(t, x) \in \mathbb{R} \times \mathbb{T}$,

$$
\partial_{t}^{2} u+z^{2}(t)\left(-\partial_{x}^{2}\right)^{\sigma} u=0, \quad u\left(t_{0}, x\right)=0, \quad \partial_{t} u\left(t_{0}, x\right)=c \exp (i|a| x),
$$

with $\sigma>0, c \in \mathbb{R}$ and $a \in \mathbb{Z}$. If $z(t)$ is non-negative and $z_{t}(t) \geq 0$, then the homogeneous energy increases, that is,

$$
\dot{\mathbb{E}}_{s}(u)(t) \geq \dot{\mathbb{E}}_{s}(u)\left(t_{0}\right) .
$$


Proof. In effect, we have the following explicit representation of the unique solution by virtue of separation of variables:

$$
u(t, x)=c y(t) \exp (i|a| x)
$$

where $y(t)$ satisfies the ordinary differential equation:

$$
y_{t t}+|a|^{2 \sigma} z^{2}(t) y=0, \quad y\left(t_{0}\right)=0, \quad y_{t}\left(t_{0}\right)=1 .
$$

By applying the definition of homogeneous Sobolev spaces $\dot{H}^{s}(\mathbb{T}), s \in \mathbb{R}$, we calculate the homogeneous energy for the solution $u=u(t, x)$. It holds by applying Lemma 2.4 .

$$
\begin{aligned}
\frac{\partial}{\partial t} \dot{\mathbb{E}}_{s}(u)(t) & =\frac{\partial}{\partial t}\left(\|u(t, \cdot)\|_{\dot{H}^{s}(\mathbb{T})}^{2}+\left\|\partial_{t} u(t, \cdot)\right\|_{\dot{H}^{s-\sigma}(\mathbb{T})}^{2}\right) \\
& =\frac{\partial}{\partial t}\left(\sum_{k \in \mathbb{Z}}|\hat{u}(t, k)|^{2}|k|^{2 s}+\sum_{k \in \mathbb{Z}}\left|\partial_{t} \hat{u}(t, k)\right|^{2}|k|^{2(s-\sigma)}\right) \\
& =\frac{\partial}{\partial t}\left(c^{2} z^{2}(t) y^{2}(t)|a|^{2 s}+c^{2} y_{t}^{2}|a|^{2(s-\sigma)}\right) \\
& =2 c^{2}\left(z z_{t} y^{2}|a|^{2 s}+z^{2} y y_{t}|a|^{2 s}+y_{t} y_{t t}|a|^{2(s \sigma)}\right) \\
& =2 c^{2} z z_{t} y^{2}|a|^{2 s} \geq 0 .
\end{aligned}
$$

Q. E. D.

From the definition of $a_{k}(t)$, it is easy to verify that $a_{k}(t)$ is monotonously increasing and differentiable in the intervals except for $I_{k}$. Therefore, according to Lemma 3.7, one has

$$
\begin{aligned}
& \dot{\mathbb{E}}_{\sigma}\left(u^{k}\right)(t) \leq \exp \left(-\varepsilon \rho_{k} h_{k}\right), \text { for } t \in\left[0, t_{k}-\rho_{k} / 2\right] ; \\
& \dot{\mathbb{E}}_{\sigma}\left(u^{k}\right)(t) \geq \exp \left(\varepsilon \rho_{k} h_{k}\right), \text { for } t \in\left[t_{k}+\rho_{k} / 2, T\right] .
\end{aligned}
$$

It is clear that (8) can be deduced immediately from (16). While for $t=t_{k}+\rho_{k} / 2$, we apply Lemma 2.4. and obtain

$$
\begin{aligned}
& \dot{\mathbb{E}}_{\sigma}\left(\exp \left(-c_{1} \nu\left(\mu^{-1}\left(2^{P_{2}} /(\sqrt{1-\Delta})^{\sigma}\right)\right)\right) u^{k}\right)(t) \\
= & \dot{\mathbb{E}}_{\sigma}\left(\exp \left(-c_{1} \nu\left(\mu^{-1}\left(2^{P_{2}} \delta_{k} / h_{k}\right)\right)\right) u^{k}\right)(t) \\
= & \exp \left(-2 c_{1} \nu\left(\mu^{-1}\left(2^{P_{2}} \delta_{k} / h_{k}\right)\right)\right) \dot{\mathbb{E}}_{\sigma}\left(u^{k}\right)(t) \\
= & \exp \left(-2 c_{1} \nu\left(\mu^{-1}\left(2^{P_{2}} \delta_{k} / h_{k}\right)\right)+\varepsilon \rho_{k} h_{k}\right) \\
= & \exp \left(-2 c_{1} \nu\left(t_{k}\right)+\varepsilon \rho_{k} h_{k}\right) .
\end{aligned}
$$

Taking into account the choice of $\rho_{k}, h_{k}$, we can choose a sufficiently small $c_{1}(\varepsilon)$ independent of $k$ such that (9) holds. This concludes the proof. Similarly, as for the non-homogeneous energy $\mathbb{E}_{s}(u)(t) \triangleq \lambda^{2}(t)\|u(t, \cdot)\|_{H^{s}(\mathbb{T})}^{2}+$ $\left\|\partial_{t} u(t, \cdot)\right\|_{H^{s-\sigma(\mathbb{T})}}^{2}, s>\sigma$, once we notice the fact (14), it is easy to check that, (8) and (9) also hold for the non-homogeneous energy $\mathbb{E}_{\sigma}(u)(t)$.

\section{Acknowledgement}

This work belongs to the following projects: 246775 - NUMERIWAVES funded by European Research Council (ERC), MTM2008-03541, funded by MICIN, ESF Research Networking Programme OPTPDE, PI2010-04, funded by Basque Government, also is partly supported by National Natural Science Foundation of China (No. 10871175). The author wishes to thank Prof. Michael Reissig from TU Bergakademie Freiberg for the fruitful discussions. Moreover, the author also expresses his deep gratitude to the referees for their careful reading and useful remarks.

\section{REFERENCES}

[1] G. Aleksandrian, Parametrix and propogation of the wave front of a solution to a Cauchy problem for a model hyperbolic equation (in Russian), Izv. Akad. Nauk. Arm. SSR 19 (1984), 33-46.

[2] F. Colombini and N. Lerner, Hyperbolic operators with non-Lipschitz coefficients, Duke Math. J. 77 (1995), $657-698$.

[3] F. Colombini, D. Del Santo and M. Reissig, On the optimal regularity of coefficients in hyperbolic Cauchy problems, Bull. Sc. Math. 1274 (2003), 328-347. 
[4] M. Cicognani and F. Colombini, Loss of derivatives in evolution Cauchy problems, Ann. Univ. Ferrara Sez. VII. Sci. Mat. 52 (2006), 271-280.

[5] M. Cicognani, F. Hirosawa and M. Reissig, The Log-effect for p-evolution type models, J. Math. Soc. Japan, 60 (2008), 819-863.

[6] M. Dreher and M. Reissig, Propagation of mild singularities for semilinear weakly hyperbolic equations, J. Anal. Math. 82 (2000), 233-266.

[7] M. Dreher, Local solutions to quasilinear weakly hyperbolic differential equations, Ph.D Thesis, Technische Universität Bergakademie Freiberg, Germany, 1999.

[8] M. R. Eberta and M. Reissig, The influence of oscillations on global existence for a class of semi-linear wave equations, Math. Meth. Appl. Sci., DOI: 10.1002/mma.1430.

[9] D. Fang, X. Lu and M. Reissig, $\nu$-loss of derivatives for an evolution type model, Nonlinear Analysis 71 (2009), 5368-5380.

[10] Lars Hörmander, The Analysis of Linear Partial Differential Operators III: Pseudo-Differential Operators, Springer, 1987.

[11] F. Hirosawa, On the Cauchy problem for second order strictly hyperbolic equations with non-regular coefficients, Math. Nach. 256 (2003), 29-47.

[12] F. Hirosawa and M. Reissig, Levi condition for hyperbolic equations with oscillating coefficients, J. Diff. Equa. 223 (2006), 329-350.

[13] F. Hirosawa, Loss of regularity for the solutions to hyperbolic equations with non-regular coefficients, an application to Kirchhoff equation, MMAS 269 (2003), 783-799.

[14] A Kubo. and M. Reissig, $C^{1}$ well-posedness of the Cauchy problem for quasi-linear hyperbolic equations with coefficients non-Lipschitz in $t$ and smooth in $x$, Banach Center Publications 60 (2003), 131-150.

[15] A. A. Kilbas, H. M. Srivastava and J. J. Trujillo, Theory and Applications of Fractional Differential Equations, Vol. 204, Elsevier Science, 2006

[16] X. Lu, On Hyperbolic/Parabolic $\sigma$-Evolution Equations, Doctoral Thesis(in Chinese), 2010.

17] X. Lu, The Applications of Microlocal Analysis in $\sigma$-Evolution Equations, Doctoral Thesis(in English), 2010.

[18] X. Lu and M. Reissig, About the precise loss of regularity for solutions to weakly hyperbolic Cauchy problems, preprint, Freiberg Dekan der Fakultät für Mathematik und Informatik, TU Bergakademie Freiberg, (2007).

[19] X. Lu and M. Reissig, Instability behavior and loss of regularity, Advances in Phase Space Analysis of Partial Differential Equations (2009), Birkhäuser Verlag, 171-200.

[20] X. Lu and M. Reissig, Does the loss of regularity really appear? Math. Meth. Appl. Sci. 32 (2009), 1246-1268.

[21] W. Magnus, S. Winkler, Hill's Equation, Interscience, 1966.

[22] S. Micu and E, Zuazua, On the controllability of a fractional order parabolic equation, Multiscale Model. Simul. 4 (2005), 36-87.

[23] M. Qi, On the Cauchy problem for a class of hyperbolic equations with initial data on the parabolic degenerating line, Acta Math. Sinica, 8 (1958), 521-529.

[24] M. Reissig, Hyperbolic equation with non-Lipschitz coefficients, Rend. Sem. Mat. Univ. Pol. Torino, Microlocal analysis, Vol. 61 (2003), 135-182

25] S. Shkoller, Notes on $L^{p}$ and Sobolev spaces, preprint, http : //www.math.ucdavis.edu/hunter $/{\text { m } 218 b_{0} 9 / \text { Lp }_{a} \text { nd }}_{S}$ obolev otes.pdf $_{\text {. }}$

[26] Z. Tu, X. Lu, Counterexamples of regularity behavior for $\sigma$-evolution equations, J. Math. Anal. Appl. 382 (2011) $148-161$.

27] Michael E. Taylor, Pseudodifferential Operators, Princeton Univ. Press, 1981.

28] S. Tarama, On the second order hyperbolic equations degenerating in the infinite order, Math. Japon. 42(3) (1995), 523-534.

29] K. Taniguchi and Y. Tozaki, A hyperbolic equation with double characteristics which has solution with branching singularities, Math.Japon., 25 (1980), 279-300. 